Supporting Information for:

\title{
Seeded Solid Phase Epitaxy of Atomic Layer Deposited Aluminum Oxide
}

Hannah Maret, Derek Weisberg, Helen M. Chan, Nicholas C. Strandwitz*

Materials Science and Engineering, Lehigh University, Bethlehem PA, 18015

Center for Advanced Materials and Nanotechnology, Lehigh University, Bethlehem PA, 18015

\section{*strand@lehigh.edu}
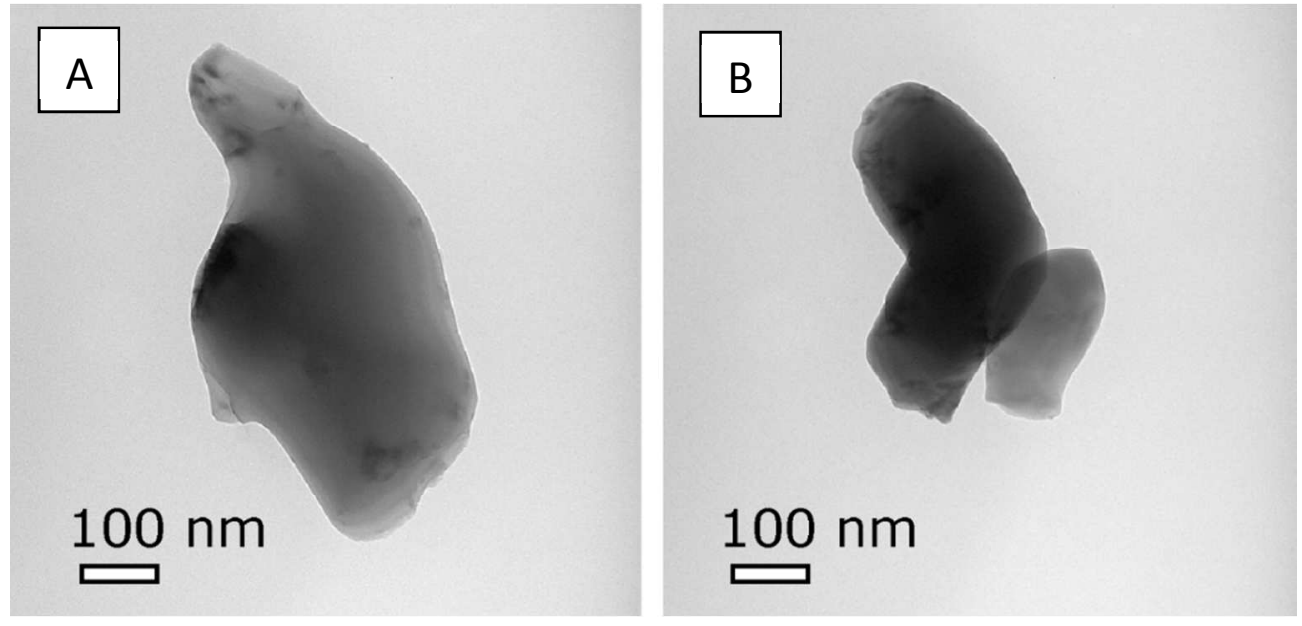

Figure S1. (A, B) TEM of two representative sapphire seed crystals.

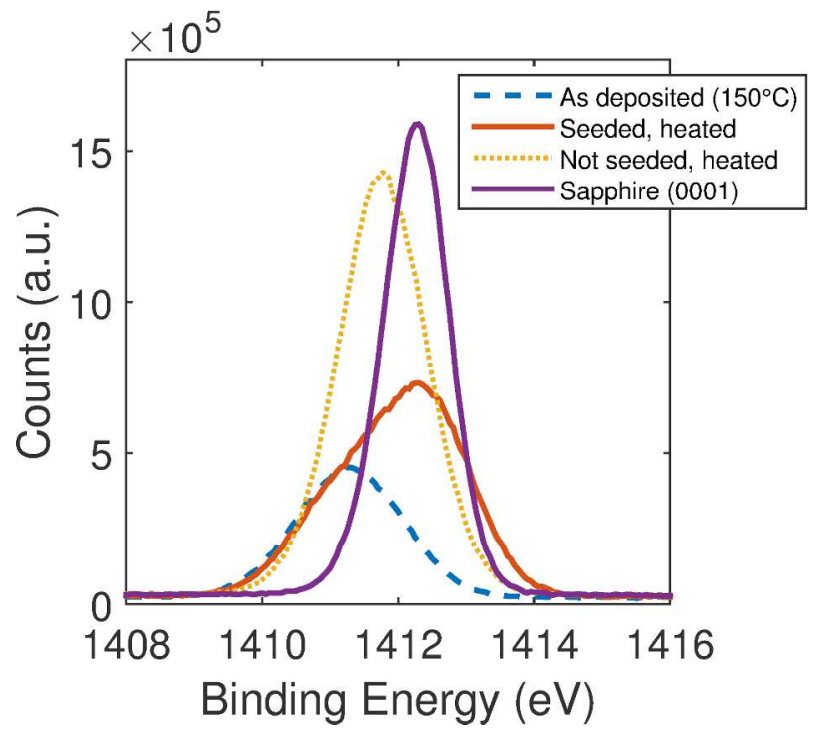

Figure S2. XPS data for Al 2p region. XP spectra were collected with monochromated Al K- $\alpha$ radiation with a $150 \mathrm{eV}$ pass energy at $50 \mathrm{meV}$ step size. Charge neutralization was used and all samples were adjusted based on the $\mathrm{C}-1 \mathrm{~s}$ peak at $284.6 \mathrm{eV}$. 


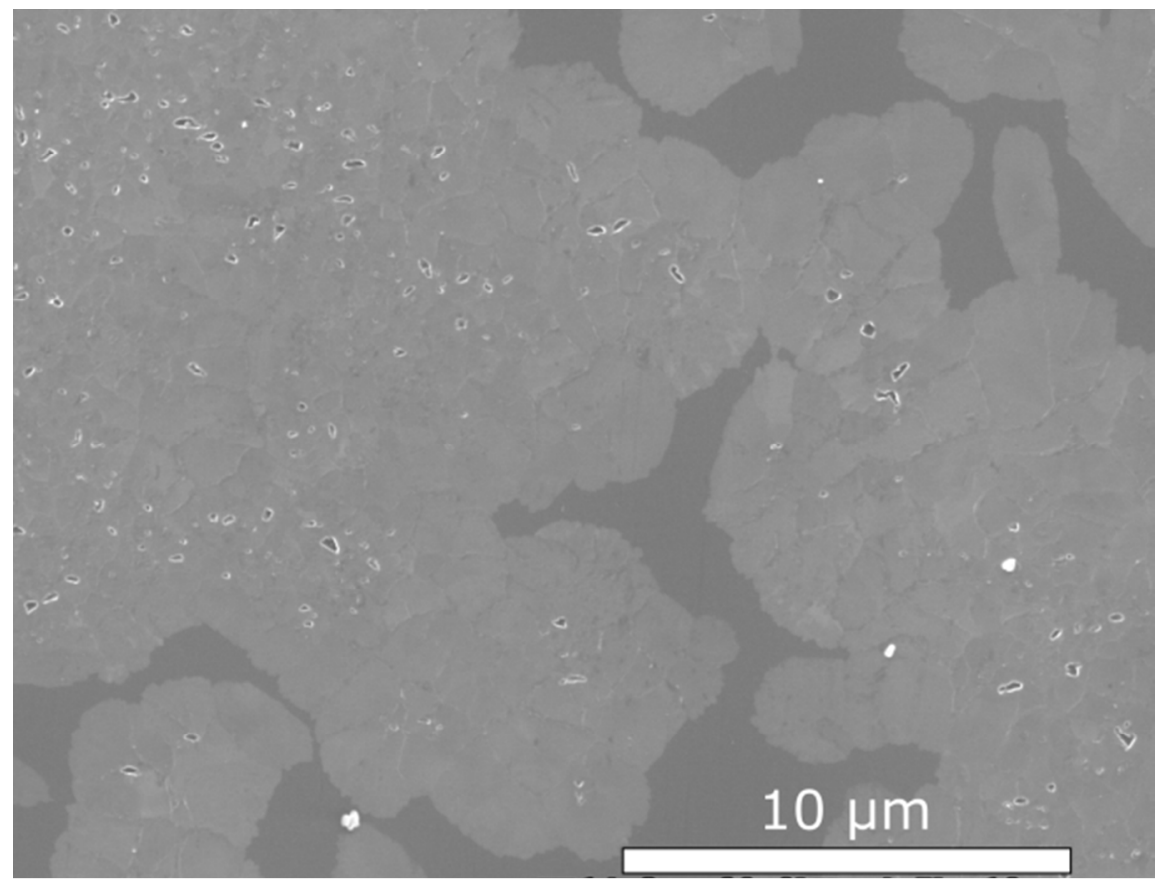

Figure S3. SEM micrograph of a seeded and crystallized film that was almost completely crystallized. The seeds were mechanically removed using a single wipe with a cloth (TechniCloth TX606). 